\title{
Contact Quantities and Non-Equilibrium Entropy of Discrete Systems*
}

\author{
Wolfgang Muschik \\ Institut für Theoretische Physik, Technische Universität Berlin, \\ Hardenbergstr. 36, 10623 Berlin, Germany \\ e-mail: muschik@physik.tu-berlin.de \\ Communicated by J.U. Keller, Siegen, Germany
}

\begin{abstract}
If a discrete non-equilibrium system is in contact with an equilibrium reservoir exchanging heat, power, and material with each other, a non-equilibrium nonadditive entropy rate of the system can be defined by use of non-equilibrium contact quantities: contact temperature and dynamic chemical potentials. The integrability of the entropy rate and the corresponding Maxwell relations are investigated. The dissipation inequality is exploited for a simple material whose state space includes the time rate of the contact temperature. A non-equilibrium efficiency is introduced.
\end{abstract}

\section{Introduction}

There are two phenomenological descriptions of classic thermodynamic systems: the field formulation portraying mainly continuous systems and the representation of heterogeneous systems in contact with each other as an ensemble of discrete systems [1]. Whereas thermodynamics of continua is a well-developed theory [2-8], the theoretical level of non-equilibrium thermodynamics of discrete systems is rather low, as remarked in the literature $[9,10]$. The reason for that is the following: In the field formulation of thermodynamics, temperature and entropy are introduced as so-called "primitive concepts" (that means, do not ask for the background), although one knows that these quantities are only well defined in equilibrium. This procedure may be acceptable for a field theory, but for the non-equilibrium theory of discrete systems,

\footnotetext{
*Partially delivered at the "Workshop on the Occasion of the 70th Anniversary of Prof. José Casas-Vázquez", 18-19 September 2008, Barcelona, and at the Thermodynamik-Kolloquium und Ingenieurdaten, 24-26 September 2008, Universität Erlangen/Nuremberg.
} 
the problem of defining temperature and entropy far from equilibrium becomes unavoidable. There is an auxiliary procedure to detour this problem, namely endoreversible thermodynamics [11]. In this frame, the discrete systems are approximately presupposed as equilibrium ones exchanging heat, power, and material with each other without disturbing their internal equilibrium.

Here, we do not use the endoreversible procedure, instead, we introduce by choosing a non-equilibrium state space an entropy rate, and a non-equilibrium temperature (the contact temperature) and the dynamic chemical potentials are defined as contact quantities [12]. It can be proved that the entropy rate and the internal energy rate are independent of each other for pure heat exchange between the non-equilibrium system and a heat reservoir. The question of whether this non-equilibrium entropy rate is integrable is answered by investigating the adiabatic uniqueness of the non-equilibrium system. The dissipation inequality is used for discussing a simple class of materials belonging to a state space which includes besides the internal energy and the contact temperature also its time rate. Finally, by using contact temperatures, a non-equilibrium efficiency is introduced which is smaller than the Carnot efficiency, thus representing a more realistic measure.

\section{Discrete systems}

\subsection{Thermostatics}

A resting discrete equilibrium system $\mathcal{G}^{*}$ is characterized by the state space

$$
Z^{*}=\left(U^{*}, \mathbf{a}^{*}, \mathbf{n}^{*}\right)
$$

spanned by its internal energy $U^{*}$, the work variables $\mathbf{a}^{*}$, and the mole numbers $\mathbf{n}^{*}$. The differential of its equilibrium entropy is given by Gibbs' fundamental equation

$$
d S^{*}=\frac{1}{T^{*}} d U^{*}-\frac{\mathbf{A}^{*}}{T^{*}} d \mathbf{a}^{*}-\frac{\boldsymbol{\mu}^{*}}{T^{*}} d \mathbf{n}^{*},
$$

introducing the generalized forces $\mathbf{A}^{*}$ and the chemical potentials $\boldsymbol{\mu}^{*}$. The equilibrium entropy $S^{*}=S^{*}\left(U^{*}, \mathbf{a}^{*}, \mathbf{n}^{*}\right)$ is a state function, and therefore the given equilibrium temperature $T^{*}$, the generalized forces $\mathbf{A}^{*}$, and the mole numbers $\mathbf{n}^{*}$ determine the partial derivatives of the equilibrium entropy

$$
\frac{\partial S^{*}}{\partial U^{*}}=\frac{1}{T^{*}}, \quad \frac{\partial S^{*}}{\partial \mathbf{a}^{*}}=-\frac{\mathbf{A}^{*}}{T^{*}}, \quad \frac{\partial S^{*}}{\partial \mathbf{n}^{*}}=-\frac{\boldsymbol{\mu}^{*}}{T^{*}} .
$$

The first law of $\mathcal{G}^{*}$ states

$$
D Q^{*}=d U^{*}-\mathbf{A}^{*} \cdot d \mathbf{a}^{*}-\mathbf{h}^{*} \cdot d \mathbf{n}^{e *} .
$$


Here $D Q^{*}$ is the "equilibrium heat exchange", $\mathbf{h}^{*}$ the molar enthalpy and $d \mathbf{n}^{e *}$ the "external equilibrium change of mole numbers". Later on, we will consider non-equilibrium states and their time rates, which will be denoted by a $\operatorname{dot}\left({ }^{\circ}\right)$. For convenience, we will replace the differentials

$$
D \boxplus^{*} \quad \text { and } \quad d \boxplus^{*} \quad \rightarrow \quad \dot{\boxplus}^{*}
$$

also by a dot, knowing that they are not time rates if they belong to equilibrium sytems: These dots are directional derivatives along the "reversible process" (a trajectory in the state space (1)). Consequently, the first law (4) states

$$
\dot{Q}^{*}=\dot{U}^{*}-\mathbf{A}^{*} \cdot \mathbf{a}^{*}-\mathbf{h}^{*} \cdot \dot{\mathbf{n}}^{e *} .
$$

This formulation of the first law and the differential (2) of the entropy

$$
\dot{S}^{*}=\frac{1}{T^{*}} \dot{U}^{*}-\frac{\mathbf{A}^{*}}{T^{*}} \cdot \dot{\mathbf{a}}^{*}-\frac{\boldsymbol{\mu}^{*}}{T^{*}} \cdot \dot{\mathbf{n}}^{*}
$$

is all that is necessary to know from the equilibrium system $\mathcal{G}^{*}$.

The time rates of the mole numbers can be split into an external ${ }^{e}$ and an internal ${ }^{i}$ part

$$
\dot{\mathbf{n}}=\dot{\mathbf{n}}^{e}+\dot{\mathbf{n}}^{i}, \quad \dot{\mathbf{n}}^{*} \equiv \dot{\mathbf{n}}^{e *}
$$

There is no internal time rate of mole numbers in $\mathcal{G}^{*}$, because chemical reactions do not take place in equilibrium ${ }^{1}$. Introducing the molar entropies

$$
\mathbf{s}^{*}:=\frac{1}{T^{*}}\left(\mathbf{h}^{*}-\boldsymbol{\mu}^{*}\right),
$$

we obtain from Eq. (7) by use of Eqs. (8) and (9)

$$
\dot{S}^{*}=\frac{\dot{Q}^{*}}{T^{*}}+\mathbf{s}^{*} \cdot \dot{\mathbf{n}}^{e *} .
$$

\subsection{Non-equilibrium}

In non-equilibrium, the state space (1) has to be extended,

$$
Z=(U, \mathbf{a}, \mathbf{n}, \Theta, \xi),
$$

\footnotetext{
${ }^{1}$ The macroscopic reaction velocity is zero in equilibrium, although the microscopic rates of the partial reactions do not vanish. According to the Guldberg-Waage law, the concentrations of the components are constant in equilibrium. If the macroscopic reaction velocity vanishes, the mole number rate $\dot{\mathbf{n}}^{i}$ is zero according to Eq. (60) below.
} 
by additional non-equilibrium variables $\Theta$ and $\xi$ characterizing the nonequilibrium state beyond the equilibrium variables. Here, $\Theta$ is a non-equilibrium temperature of the system $\mathcal{G}$, which later on will be identified with the contact temperature [13-15]. The $\xi$ are other non-equilibrium variables which do not need to be characterized in more detail at the present stage of discussion [16]. How to define the contact temperature by a measuring procedure is discussed later on in Section 4. For the present, we will take $\Theta$ as an unknown placeholder.

Now we define the time rate of a non-equilibrium entropy of $\mathcal{G}$. According to Eq. (11), the non-equilibrium time rate of entropy becomes

$$
\dot{S}:=\frac{1}{\Theta} \dot{U}-\frac{\mathbf{A}}{\Theta} \cdot \dot{\mathbf{a}}-\frac{\boldsymbol{\mu}}{\Theta} \cdot \dot{\mathbf{n}}+\alpha \dot{\Theta}+\boldsymbol{\beta} \cdot \dot{\boldsymbol{\xi}} .
$$

A comparison with the time rate of the equilibrium entropy (7) shows that the equilibrium temperature $T$ is replaced by the as yet undefined temperature $\Theta$; the equilibrium quantities $\mathbf{A}^{*}$ and $\boldsymbol{\mu}^{*}$ are substituted by non-equilibrium quantities $\mathbf{A}$ and $\boldsymbol{\mu}$. Additionally, $\alpha$ and $\boldsymbol{\beta}$ appear, which have no analogues in equilibrium, because $\Theta$ and $\boldsymbol{\xi}$ do not appear in $Z^{*}$. For the present, the physical meaning of $\Theta, \boldsymbol{\mu}, \alpha, \boldsymbol{\beta}$, and $\boldsymbol{\xi}$ remains open. Additionally, we will not presuppose that the time rate of the non-equilibrium entropy $\dot{S}$ is the time derivative of a state function $S(U, \mathbf{a}, \mathbf{n}, \Theta, \xi)$. Consequently, at the present stage of discussion we do not have in non-equilibrium integrability conditions analogous to Eq. (3) .

Analogously to Eq. (6), the first law of $\mathcal{G}$ states

$$
\dot{Q}=\dot{U}-\mathbf{A} \cdot \dot{\mathbf{a}}-\mathbf{h} \cdot \dot{\mathbf{n}}^{e} .
$$

Analogously to Eq. (8), we define the molar entropy in non-equilibrium as

$$
\mathbf{s}:=\frac{1}{\Theta}(\mathbf{h}-\boldsymbol{\mu}) \text {. }
$$

By the use of Eqs. (8) and (14), the entropy rate in non-equilibrium states (12) becomes

$$
\dot{S}=\frac{1}{\Theta}\left[\dot{Q}+\mathbf{h} \cdot \dot{\mathbf{n}}^{e}\right]-\frac{\boldsymbol{\mu}}{\Theta} \cdot \dot{\mathbf{n}}+\alpha \dot{\Theta}+\boldsymbol{\beta} \cdot \dot{\xi} .
$$

The entropy rates (10) and (15) can be split into the entropy exchanges

$$
\Phi^{*}:=\frac{\dot{Q}^{*}}{T^{*}}+\mathbf{s}^{*} \cdot \dot{\mathbf{n}}^{e *}=\dot{S}^{*}, \quad \Phi:=\frac{\dot{Q}}{\Theta}+\mathbf{s} \cdot \dot{\mathbf{n}}^{e}=\dot{S}-\Sigma,
$$

and into the entropy production in $\mathcal{G}$,

$$
\Sigma:=-\frac{\boldsymbol{\mu}}{\Theta} \cdot \dot{\mathbf{n}}^{i}+\alpha \dot{\Theta}+\boldsymbol{\beta} \cdot \dot{\boldsymbol{\xi}} \geq 0,
$$


by isolating $\mathcal{G}$ and $\mathcal{G}^{*}$ : The entropy rate of an isolated system is by definition its entropy production. As expected, Eq. (16) 1 shows that there is no entropy production in equilibrium systems, so that in such systems the entropy exchange is equal to its entropy rate.

In Eq. (17) it is presupposed that $\dot{\Theta}$ and $\dot{\xi}$ are quantities which are not influenced by isolating $\mathcal{G}$, as obviously the internal change of mole numbers due to chemical reactions $\dot{\mathbf{n}}^{i}$ are not. In contrast to $\dot{\Theta}$ and $\dot{\xi}$, the exchange terms $\dot{Q}$ and $\dot{\mathbf{n}}^{e}$ in Eq. (16) 2 are set to zero by isolating $\mathcal{G}$. The inequality in Eq. (17) stems from the strong formulation of the second law for $\mathcal{G}$ [17].

\section{Compound system}

We consider an isolated compound (or composite) system [17] which consists of two subsystems which are in contact with each other: One of them is "the system $\mathcal{G}$ " being in non-equilibrium, the other one is a reservoir in equilibrium marked by * and is called "the environment $\mathcal{G}^{*}$ " of the considered non-equilibrium system $\mathcal{G}$. Both are separated by the bounded surface $\partial \mathcal{G}$ enclosing the system $\mathcal{G}$. This surface $\partial \mathcal{G}$ allows exchanges of power, mass, and heat between $\mathcal{G}$ and its equilibrium environment $\mathcal{G}^{*}$. Because the environment is presupposed to be a reservoir, it is always in equilibrium, also during the contact between $\mathcal{G}$ and $\mathcal{G}^{*}$. Consequently, $\mathcal{G}^{*}$ can be described by the tools of thermostatics and its entropy is given by Eq. (10), whereas that of $\mathcal{G}$ is Eq. (15).

\subsection{Defining inequalities}

Now we have to define an entropy rate for the compound system $\mathcal{G}^{*} \cup \mathcal{G}$, consisting of $\mathcal{G}$ and $\mathcal{G}^{*}$ which are contacted by $\partial \mathcal{G}$. If we presuppose that the composite system is an isolated one, we know according to the strong formulation of the second law that its entropy rate is equal to its non-negative entropy production. Summing up Eqs. (16) and (17), we obtain with Eq. (15)

$$
\dot{S}^{*}+\dot{S}=\Phi^{*}+\Phi+\Sigma .
$$

From Eqs. (16), (9), and (14) follows

$$
\begin{aligned}
\Phi^{*}+\Phi= & \frac{1}{T^{*}}\left[\dot{Q}^{*}+\mathbf{h}^{*} \cdot \dot{\mathbf{n}}^{e *}\right]- \\
& -\frac{\mu^{*}}{T^{*}} \cdot \dot{\mathbf{n}}^{e *}+\frac{1}{\Theta}\left[\dot{Q}+\mathbf{h} \cdot \dot{\mathbf{n}}^{e}\right]-\frac{\mu}{\Theta} \cdot \dot{\mathbf{n}}^{e} .
\end{aligned}
$$


This equation is invariant under changing the star quantities of $\mathcal{G}^{*}$ with the unstarred quantities of $\mathcal{G}$.

An inert partition, one which does not emit or absorb heat nor particles, is defined by $[18,19]$

$$
\dot{Q}^{*}+\mathbf{h}^{*} \cdot \dot{\mathbf{n}}^{e *}=-\dot{Q}-\mathbf{h} \cdot \dot{\mathbf{n}}^{e}, \quad \dot{\mathbf{n}}^{e *}=-\dot{\mathbf{n}}^{e} .
$$

Consequently, for an inert partition Eq. (19) results in

$$
\Phi^{*}+\Phi=\left(\frac{1}{T^{*}}-\frac{1}{\Theta}\right)\left[\dot{Q}^{*}+\mathbf{h}^{*} \cdot \dot{\mathbf{n}}^{e *}\right]+\left(\frac{\boldsymbol{\mu}}{\Theta}-\frac{\mu^{*}}{T^{*}}\right) \cdot \dot{\mathbf{n}}^{e *} .
$$

The invariance of changing $\mathcal{G}^{*}$ with $\mathcal{G}$ remains also valid for Eq. (21). We now use Eq. (20) again:

$$
\begin{aligned}
\Phi^{*}+\Phi= & \frac{1}{2}\left(\frac{1}{T^{*}}-\frac{1}{\Theta}\right)\left[\dot{Q}^{*}+\mathbf{h}^{*} \cdot \dot{\mathbf{n}}^{e *}-\dot{Q}-\mathbf{h} \cdot \dot{\mathbf{n}}^{e}\right]+ \\
& +\frac{1}{2}\left(\frac{\mu}{\Theta}-\frac{\boldsymbol{\mu}^{*}}{T^{*}}\right) \cdot\left(\dot{\mathbf{n}}^{e *}-\dot{\mathbf{n}}^{e}\right) .
\end{aligned}
$$

As shown in the appendix, a short calculation taking Eqs. (9) and (14) into account results in

$$
\begin{aligned}
& \Phi^{*}+\Phi-\frac{1}{2}\left(\frac{1}{T^{*}}-\frac{1}{\Theta}\right)\left[T^{*} \mathbf{s}^{*}+\Theta \mathbf{s}\right] \cdot \dot{\mathbf{n}}^{e *}= \\
& =\frac{1}{2}\left(\frac{1}{T^{*}}-\frac{1}{\Theta}\right)\left(\dot{Q}^{*}-\dot{Q}\right)+\frac{1}{2}\left(\frac{1}{T^{*}}+\frac{1}{\Theta}\right)\left(\boldsymbol{\mu}-\boldsymbol{\mu}^{*}\right) \cdot \dot{\mathbf{n}}^{e *} .
\end{aligned}
$$

The invariance of changing $\mathcal{G}^{*}$ with $\mathcal{G}$ is still valid for Eq. (23).

We recall that the contact temperature $\Theta$ and the chemical potentials $\mu$ are as yet only placeholders which we now will define by two inequalities, called the defining inequalities of $\Theta$ and $\boldsymbol{\mu}$ whose interpretation will be discussed in detail in the next section:

$$
\begin{array}{r}
\left(\frac{1}{T^{*}}-\frac{1}{\Theta}\right)\left[\dot{Q}^{*}-\dot{Q}\right] \geq 0, \\
\left(\boldsymbol{\mu}-\boldsymbol{\mu}^{*}\right) \cdot \dot{\mathbf{n}}^{e *} \geq 0 .
\end{array}
$$

The latter should be valid for each of the $K$ components in $\mathcal{G}$,

$$
\left(\mu_{j}-\mu_{j}^{*}\right) \dot{n}_{j}^{e *} \geq 0, \quad j=1,2, \ldots, K .
$$




\subsection{Total entropy rate}

According to Eqs. (17) and (18), the defining inequalities (24) and (25) enforce that the second law is satisfied for the isolated composite system, if the total entropy rate is defined as follows:

$$
\dot{S}^{t o t}:=\dot{S}^{*}+\dot{S}-\frac{1}{2}\left(\frac{1}{T^{*}}-\frac{1}{\Theta}\right)\left[T^{*} \mathbf{s}^{*}+\Theta \mathbf{s}\right] \cdot \dot{\mathbf{n}}^{e *} \geq 0 .
$$

The entropy rates of $\mathcal{G}^{*}$ and $\mathcal{G}$ are only additive, if there is no material exchange between system and environment and/or the contact temperature of the system is equal to the thermostatic temperature of the environment.

It is possible to avoid a non-additive total entropy rate: If in the formulations of the first law (6) and (13) the molar enthalpies $\mathbf{h}^{*}$ and $\mathbf{h}$ are replaced by the chemical potentials $\boldsymbol{\mu}^{*}$ and $\boldsymbol{\mu}$, the external mole number rates $\dot{\mathbf{n}}^{e *}$ and $\dot{\mathbf{n}}^{e}$ do not appear in Eqs. (10) and (15). Formally, in this case the molar entropies $\mathbf{s}^{*}$ and $\mathbf{s}$ have to be set to zero and, consequently, the entropy exchanges (16) are defined without a part belonging to the material exchange. Although the total entropy rate becomes additive in this case, according to Eq. (27), we will not pursue this path, because the entropy exchange coupled to the material exchange is ignored.

Another consequence of the defining inequalities (24) and (25) is that the entropy exchange through inert partitions is discontinuous in contrast to the combined material and heat exchanges $(20)_{1}$. From Eq. (23) we obtain

$$
\Phi^{*}+\Phi \geq \frac{1}{2}\left(\frac{1}{T^{*}}-\frac{1}{\Theta}\right)\left[T^{*} \mathbf{s}^{*}+\Theta \mathbf{s}\right] \cdot \dot{\mathbf{n}}^{e *} .
$$

Continuity of the entropy exchange enforces the equality in Eq. (28) and that $\Theta=T^{*}$ and/or $\mathbf{n}^{e *}=\mathbf{0}$ are valid. We now will investigate whether the entropy exchange can be continuous in one of these two cases.

First of all, we need the following proposition [20]:

$$
\mathbf{X} \cdot \mathbf{f}(\mathbf{X}) \geq 0 \text {, for all } \mathbf{X} \wedge \mathbf{f} \text { continuous at } \mathbf{X}=\mathbf{0} \Rightarrow \mathbf{f}(\mathbf{0})=\mathbf{0} .
$$

Starting out with $\Theta=T^{*}$ and applying Eq. (29) to the defining inequality (24), we obtain

$$
\dot{Q}^{*}-\dot{Q}=0 \quad \longrightarrow \quad \dot{Q}^{*}=0 \text { and } \dot{Q}=0,
$$

because $\operatorname{sign} \dot{Q}^{*}=-\operatorname{sign} \dot{Q}$ is valid. Consequently, the entropy exchanges (16) become

$$
\Phi^{*}+\Phi=\mathbf{s} \cdot \dot{\mathbf{n}}^{e}+\mathbf{s}^{*} \cdot \dot{\mathbf{n}}^{e *} \neq 0
$$


Thus, the entropy exchange is discontinuous, if $\mathbf{s} \neq \mathbf{s}^{*}$.

Starting out with $\dot{\mathbf{n}}^{e *}=\mathbf{0}=-\dot{\mathbf{n}}^{e}$, the entropy exchanges (16) become

$$
\Phi^{*}+\Phi=\frac{\dot{Q}^{*}}{T^{*}}+\frac{\dot{Q}}{\Theta} \geq 0,
$$

because the inequality follows according to Eq. (20) and the defining inequality (24). Also in this case, the entropy exchange is not continuous. That means, the entropy exchange at inert partitions is only continuous and zero in equilibrium.

The result of this section is astonishing: Also in classical thermodynamics, the total entropy rate is non-additive in general. The reason for this fact is that the entropy exchanges (16) should be formulated with the correct exchange term due to material exchange. According to Eq. (27), the total entropy rate becomes additive in the special cases of equal temperatures $\Theta=T^{*}$, that is, vanishing heat exchange, and of $\dot{\mathbf{n}}^{e *}=\mathbf{0}$, that is, vanishing material exchange between the system and its environment. The entropy exchange is discontinuous in any case, except that of equilibrium. Of course, all these statements depend on a satisfying interpretation of the defining inequalities (24) and (25), which will be given in the next section.

\section{Contact quantities}

Recall what we are considering a discrete non-equilibrium system in contact with an equilibrium (reservoir) environment exchanging heat, power, and material. For describing the non-equilibrium system, we introduced in Eq. (12) two nonequilibrium quantities, a temperature $\Theta$, and a chemical potential $\boldsymbol{\mu}$, as place holders whose physical meaning has remained open up to now. For defining the total entropy rate (27) and the entropy exchange (23), two inequalities (24) and (25), the defining inequalities are presupposed which define the $\Theta$ and $\mu$, as dicussed in the following section.

\subsection{Contact temperature}

The defining inequalitiy of $\Theta$ (24) in connection with the proposition (29) and taking $\operatorname{sign} \dot{Q}^{*}=-\operatorname{sign} \dot{Q}$ into account yields

$$
\begin{aligned}
& T^{*}>\Theta \Longleftrightarrow \dot{Q}^{*}-\dot{Q}<0 \Longrightarrow \dot{Q}^{*}<0, \dot{Q}>0, \\
& T^{*}<\Theta \Longleftrightarrow \dot{Q}^{*}-\dot{Q}>0 \Longrightarrow \dot{Q}^{*}>0, \dot{Q}<0 .
\end{aligned}
$$


What is the heat exchange between $\mathcal{G}$ and $\mathcal{G}^{*}$, if $T^{*}=\Theta$ ? To answer this question, we presuppose the following:

\section{Axiom I}

For inert partitions, the heat exchange $\dot{Q}^{*}-\dot{Q}$ between open systems is a continuous function of the temperature difference $\left[\Theta-T^{*}\right]$.

According to Eq. (29), we obtain

$$
\Theta=T^{*} \Longleftrightarrow \dot{Q}^{*}=0=\dot{Q} .
$$

Consequently, the inequality (24) is the

\section{Definition}

of the contact temperature $\Theta[21]$ as that thermostatic temperature of the environment $\mathcal{G}^{*}$ for which the net heat exchange between the open systems $\mathcal{G}$ and $\mathcal{G}^{*}$ through an inert partition $\partial \mathcal{G}$ vanishes by undergoing a change of its sign [19].

This definition makes it possible to measure the contact temperature by calorimetry.

We will now demonstrate that the contact temperature $\Theta$ and the internal energy $U$ are independent of each other. For this purpose, we choose a rigid inert partition $(\mathbf{a}=\mathbf{0})$ which is impervious to matter $\left(\dot{\mathbf{n}}^{e}=\mathbf{0}\right)$ and a timedependent environment temperature $T^{*}(t)$ which is always equal to the value of the momentary contact temperature of $\mathcal{G}$ :

$$
T^{*}(t) \doteq \Theta(t) \Longrightarrow \dot{Q}^{*}(t)=\dot{Q}=0 \Longrightarrow \dot{U}^{*}(t)=\dot{U}(t)=0 .
$$

The last implication is due to the first law (6) and (13) of closed systems without power exchange. Because $\Theta$ is time-dependent and $U$ constant, both quantities are independent of each other.

\subsection{Dynamic chemical potentials}

We now consider the defining inequality (25) for $\boldsymbol{\mu}$. Analogously to axiom I, we state:

\section{Axiom II}

For inert partitions, the $\dot{\mathbf{n}}^{e *}$ are continuous functions of the differences of the molar entropies $\left[\boldsymbol{\mu}-\boldsymbol{\mu}^{*}\right]$.

Analogously to Eq. (35), we obtain from Eq. (25) and axiom II by applying Eq. (29)

$$
\mu=\mu^{*} \quad \Longrightarrow \quad \dot{\mathbf{n}}^{e *}=\mathbf{0} .
$$


Consequently, the inequality (25) is the

Definition

of the dynamic chemical potentials $\boldsymbol{\mu}$ of $\mathcal{G}$ as those chemical potentials $\boldsymbol{\mu}^{*}$ of the environment $\mathcal{G}^{*}$ which cause vanishing external mole number exchanges under change of their signs [21].

\section{Adiabatical uniqueness}

The integrability conditions (3) of the equilibrium entropy of $\mathcal{G}^{*}$ are for the present not satisfied for the non-equilibrium entropy rate (12): There is no state function non-equilibrium entropy defined on the state space (11). One reason for that may be that the state space is a small one [21,22] and consequently the non-equilibrium entropy depends on the history of the processes on Eq. (11). All consideration about contact quantities made above are also valid in small state spaces, because no integrability conditions are necessary for defining contact quantities.

We now ask the question: What conditions are necessary so that the nonequilibrium entropy rate (12) satisfies integrability expressions analogously to Eq. (3)? We consider an arbitrary system undergoing a family of processes all starting out from an equilibrium state $A^{e q}$ and going to a non-equilibrium state $B$ :

$$
\mathcal{C}: A^{e q} \longrightarrow B, \quad \text { for arbitrary } \mathcal{C} .
$$

Arriving at $B$, the system in consideration is isolated and reaches an equilibrium state $C^{e q}$ :

$$
\mathcal{I}: \quad B \longrightarrow C^{e q}, \quad\left(\dot{Q}=0, \dot{W}=0, \dot{\mathbf{n}}^{e}=\mathbf{0}\right) .
$$

We now give the

\section{Definition}

A system is called adiabatically unique, if for each arbitrary, but fixed non-equilibrium state $B$ after isolation of the system, the process $\mathcal{I}$ ends always in the same final equilibrium state $C^{e q}$, independently of how the process $\mathcal{C}$ to $B$ was performed.

For exploiting the adiabatical uniqueness of a system, we have to remember the embedding axiom of non-equilibrium thermodynamics [23]:

\section{Axiom III}

The non-equilibrium entropy rate has to be in accordance with the equilibrium entropy: 


$$
\mathcal{T} \int_{A^{e q}}^{C^{e q}} \dot{S} d t=S_{C}^{e q}-S_{A}^{e q}, \quad \text { for arbitrary } \mathcal{T} \text { between } A^{e q} \text { and } C^{e q} .
$$

We now specify the arbitrary process $\mathcal{T}$ in Eq. (40)

$$
\mathcal{T}=\mathcal{C} \cup \mathcal{I} \Longrightarrow \mathcal{C} \int_{A^{e q}}^{B} \dot{S} d t+\mathcal{I} \int_{B}^{C^{e q}} \dot{S} d t=S_{C}^{e q}-S_{A}^{e q},
$$

by the successive performance of the processes defined in Eqs. (38) and (39) which are used in the definition of adiabatical uniqueness. If adiabatical uniqueness is now presupposed, $C^{e q}$ is unique, is determined by $B$, and is independent of all allowed $\mathcal{C}$ according to Eq. (38). Then Eq. (41) results in

$$
\mathcal{C} \int_{A^{e q}}^{B} \dot{S} d t+S_{C}^{e q}-S_{B}=S_{C}^{e q}-S_{A}^{e q}, \quad \text { for all } \mathcal{C} .
$$

Consequently, the integral in Eq. (42) is path independent. Introducing its value $S_{B}-S_{A}^{e q}$ into Eq. (41), we obtain

$$
S_{B}=S_{C}^{e q}-\mathcal{I} \int_{B}^{C^{e q}} \dot{S} d t,
$$

which is the definition of a non-equilibrium entropy (and not of a rate!) of $B$. Therefore, we proved the

\section{Proposition}

If a system $\mathcal{G}$ is adiabatically unique, a non-equilibrium entropy exists

$$
S=S(U, \mathbf{a}, \mathbf{n}, \Theta, \boldsymbol{\xi}),
$$

and the path integrals on the state space over the entropy rate are path independent.

\section{Integrability conditions}

If the system $\mathcal{G}$ is adiabatically unique, a comparison between Eqs. (44) and (12) results in the integrability conditions

$$
\begin{array}{r}
\frac{\partial S}{\partial U}=\frac{1}{\Theta}, \quad \frac{\partial S}{\partial \mathbf{a}}=-\frac{\mathbf{A}}{\Theta}, \quad \frac{\partial S}{\partial \mathbf{n}}=-\frac{\boldsymbol{\mu}}{\Theta}, \\
\frac{\partial S}{\partial \Theta}=\alpha, \quad \frac{\partial S}{\partial \boldsymbol{\xi}}=\boldsymbol{\beta},
\end{array}
$$

which are analogous to those in equilibrium (3). Because $\Theta$ and $U$ are independent of each other according to Eq. (36), we can integrate Eq. (45) immediately:

$$
S(U, \mathbf{a}, \mathbf{n}, \Theta, \boldsymbol{\xi})=\frac{1}{\Theta} U+K(\mathbf{a}, \mathbf{n}, \Theta, \boldsymbol{\xi}) .
$$


Consequently, the non-equilibrium entropy is a linear function of the internal energy. Here,

$$
-\Theta K=F(\mathbf{a}, \mathbf{n}, \Theta, \boldsymbol{\xi})
$$

is the free energy $F$ of $\mathcal{G}$. In the case of equilibrium, the non-equilibrium variables of the state space (11) become dependent on the equilibrium variables. Thus, Eq. (47) becomes in equilibrium by taking Eq. (48) into account:

$$
\Theta^{e q} S^{e q}=U^{e q}-F\left(\mathbf{a}^{e q}, \mathbf{n}^{e q}, \Theta\left(U^{e q}, \mathbf{a}^{e q}, \mathbf{n}^{e q}\right), \boldsymbol{\xi}\left(U^{e q}, \mathbf{a}^{e q}, \mathbf{n}^{e q}\right)\right),
$$

an expression which is in contrast to Eq. (47) non-linear in $U^{e q}$.

From the integrability conditions (45) and (46) follows that, except for $\alpha$, all constitutive equations do not depend on the internal energy $U$ :

$$
\begin{aligned}
& \frac{\partial}{\partial \mathbf{a}} \frac{\partial S}{\partial U}=\mathbf{0} \quad \Longrightarrow \quad \frac{\partial \mathbf{A}}{\partial U}=\mathbf{0}, \\
& \frac{\partial}{\partial \mathbf{n}} \frac{\partial S}{\partial U}=\mathbf{0} \quad \Longrightarrow \quad \frac{\partial \boldsymbol{\mu}}{\partial U}=\mathbf{0}, \\
& \frac{\partial}{\partial \boldsymbol{\xi}} \frac{\partial S}{\partial U}=\mathbf{0} \Longrightarrow \frac{\partial \boldsymbol{\beta}}{\partial U}=\mathbf{0}, \\
& \frac{\partial}{\partial \Theta} \frac{\partial S}{\partial U}=-\frac{1}{\Theta^{2}}=\frac{\partial \alpha}{\partial U} .
\end{aligned}
$$

Differentiating Eq. (47) and comparison with Eqs. (45) and (46) result for the free energy (48) in

$$
\frac{\partial F}{\partial \Theta}=-S-\Theta \alpha, \quad \frac{\partial F}{\partial \mathbf{a}}=\mathbf{A}, \quad \frac{\partial F}{\partial \mathbf{n}}=\boldsymbol{\mu}, \quad \frac{\partial F}{\partial \boldsymbol{\xi}}=-\Theta \boldsymbol{\beta} .
$$

According to Eq. (54) $)_{1}$, the constitutive mapping $\alpha$ is as well linear in the internal energy as the non-equilibrium entropy (47).

\section{Dissipation inequality}

There are three inequalities: the dissipation inequality (17), the defining inequality of the contact temperature (24), and that of the dynamic chemical potentials (25). The latter inequalities contain the intensive variables $T^{*}$ and $\mu^{*}$ of the environment of the system. Consequently, the exchange quantities $\dot{Q}$ and $\dot{\mathbf{n}}^{e}$ depend also on the state of the environment, and therefore these exchange quantities cannot be included in the state space (11). 
First of all, we want to fix a special state space by choosing the variables $\xi$. Here we specify

$$
\xi \doteq \dot{\Theta}
$$

Consequently, the state space (11) becomes

$$
Z=(U, \mathbf{a}, \mathbf{n}, \Theta, \dot{\Theta}),
$$

and Eq. (47) results in

$$
S(U, \mathbf{a}, \mathbf{n}, \Theta, \dot{\Theta})=\frac{1}{\Theta} U-\frac{F}{\Theta}(\mathbf{a}, \mathbf{n}, \Theta, \dot{\Theta}) .
$$

The so-called higher derivatives of the state space (56) are [24]

$$
\mathbf{y}=\left(\dot{U}, \dot{\mathbf{a}}, \dot{\mathbf{n}}^{i}, \dot{\mathbf{n}}^{e}, \ddot{\Theta}\right) .
$$

Presupposing the state space (56), the entropy production (17) results in

$$
\Sigma=-\frac{\mu}{\Theta} \cdot \dot{\mathbf{n}}^{i}+\alpha \dot{\Theta}+\lambda \ddot{\Theta} \geq 0, \quad-\lambda:=\frac{1}{\Theta} \frac{\partial F}{\partial \dot{\Theta}}=-\frac{\partial S}{\partial \dot{\Theta}} .
$$

Here Eqs. (54) 4 and (47) yield Eq. (59) 2 . The constitutive equations $\mu, \alpha$, and $\lambda$ depend on the state space variables (56), and therefore they are independent of the higher derivatives $\mathbf{y}$ which are outside of the state space (56). Two rates in the entropy production $(59)_{1}, \dot{\mathbf{n}}^{i}$ and $\ddot{\Theta}$, are higher derivatives.

If the higher derivatives do not depend on the state space variables by balance type equations, their values can be chosen local arbitrarily. By this possible choice, we could destroy the dissipation inequality (59) ${ }_{1}$. Consequently, the factor belonging to the higher derivative has to be set to zero in this case, and the considered term does not appear in the entropy production. Here, both the higher derivatives in Eq. $(59)_{1}$ depend on the state variables, as we will demonstrate below.

The internal rates of the mole numbers due to chemical reactions can be represented by the reaction velocities $\dot{\kappa}[25]$ :

$$
\dot{\mathbf{n}}^{i}=\underline{\underline{\boldsymbol{v}}} \cdot \dot{\boldsymbol{\kappa}} .
$$

Here $\underline{\underline{v}}$ is the $k r$-matrix of the stochiometric coefficients of $r$ reactions between $k$ components. Therefore we obtain

$$
-\frac{\mu}{\Theta} \cdot \dot{\mathbf{n}}^{i}=-\frac{\mu}{\Theta} \cdot \underline{\underline{v}} \cdot \dot{\kappa}=: \frac{1}{\Theta} \mathcal{A} \cdot \dot{\kappa}
$$


with the affinities $\mathcal{A}$ of the $r$ reactions in $\mathcal{G}$ defined by

$$
\mathcal{A}:=-\mu \cdot \underline{\underline{v}} .
$$

Because the reaction velocities $\dot{\kappa}$ depend on the affinities $\mathcal{A} / \Theta[25]$,

$$
\dot{\boldsymbol{\kappa}}=\mathbf{f}\left(\frac{\mathcal{A}}{\Theta}\right), \quad \mathbf{f}(\mathbf{0})=\mathbf{0},
$$

we obtain from Eqs. (60) and (45) 3 relaxation equations

$$
\dot{\mathbf{n}}^{i}=\underline{\underline{\boldsymbol{v}}} \cdot \mathbf{f}\left(\frac{\mathcal{A}}{\Theta}\right)=\underline{\underline{\boldsymbol{v}}} \cdot \mathbf{f}\left(\frac{\partial S}{\partial \mathbf{n}} \cdot \underline{\underline{\boldsymbol{v}}}\right) .
$$

Applying this procedure, we have replaced the internal change of mole numbers $\dot{\mathbf{n}}^{i}$ by the reaction velocities $\dot{\boldsymbol{\kappa}}$, and the chemical potentials $\boldsymbol{\mu}$ by the affinities $\mathcal{A}$. The dissipation inequality $(59)_{1}$ results in

$$
\Sigma=\frac{1}{\Theta} \mathcal{A} \cdot \dot{\boldsymbol{\kappa}}+\frac{\partial S}{\partial \Theta} \dot{\Theta}+\frac{\partial S}{\partial \dot{\Theta}} \ddot{\Theta} \geq 0,
$$

if Eqs. (46) $)_{1}$ and (59) 2 are taken into account.

Applying Eq. (29) to Eq. (65) and taking Eq. (63) into account, we see that $\dot{\boldsymbol{\kappa}}$, $\dot{\Theta}$, and $\Theta$ are homogeneous in $\mathcal{A} / \Theta, \partial S / \partial \Theta$, and $\partial S / \partial \dot{\Theta}$ :

$$
\left(\begin{array}{c}
\dot{\boldsymbol{\kappa}} \\
\dot{\Theta} \\
\ddot{\Theta}
\end{array}\right)=\left(\begin{array}{ccc}
\underline{\mathbf{b}} & \mathbf{0} & \mathbf{0} \\
\overline{\mathbf{k}} & l & m \\
\mathbf{0} & p & q
\end{array}\right)\left(\begin{array}{c}
\mathcal{A} / \Theta \\
\partial S / \partial \Theta \\
\partial S / \partial \dot{\Theta}
\end{array}\right) .
$$

The constitutive mappings $\underline{\mathbf{b}}(Z), \mathbf{k}(Z), l(Z), m(Z), \mathbf{o}(Z), p(Z)$, and $q(Z)$ are defined on the state space $(\overline{\overline{56}})$. The matrix equation (66) is not a linear mapping, but describes the homogeneity relation due to Eq. (65). The zeros of the matrix in Eq. (66) result from Eq. (63).

If

$$
\frac{\partial S}{\partial \Theta}=0 \wedge \frac{\partial S}{\partial \dot{\Theta}}=0 \Longrightarrow \frac{1}{\Theta} \mathcal{A} \cdot \dot{\kappa} \geq 0
$$

follows from Eq. (65). Because $\dot{\boldsymbol{\kappa}}$ and $\mathcal{A} / \Theta$ do not depend on $\partial S / \partial \Theta$ and $\partial S / \partial \dot{\Theta}$ according to Eq. (66), the inequality (67) is valid in general. Consequently, we obtain by Eq. (66) that the constitutive mapping $\underline{\underline{\mathbf{b}}}$ is positive semi-definitely:

$$
\mathcal{A} \cdot \underline{\underline{\mathbf{b}}} \cdot \mathcal{A} \geq 0
$$


The two last rows of Eq. (66) are

$$
\begin{aligned}
& \dot{\Theta}=\mathbf{k}(Z) \cdot(\mathcal{A} / \Theta)+l(Z) \frac{\partial S}{\partial \Theta}+m(Z) \frac{\partial S}{\partial \dot{\Theta}}, \\
& \ddot{\Theta}=\mathbf{o}(Z) \cdot(\mathcal{A} / \Theta)+p(Z) \frac{\partial S}{\partial \Theta}+q(Z) \frac{\partial S}{\partial \dot{\Theta}} .
\end{aligned}
$$

These two equations are of different meaning: Eq. (69) is a constraint for the constitutive equations and the state space variable $\dot{\Theta}$. In contrast to Eq. (69), the second equation (70) is a relaxation type equation of the higher derivative $\ddot{\Theta}$. Consequently, by this relaxation equation the higher derivative $\ddot{\Theta}$ becomes a function of the independent basic state space variables.

The choice of the state space (56), the resulting dissipation inequality (65), and the homogeneity conditions (66) define a class of materials which is characterized by all the constitutive equations which are compatible with the first law (13), with the dissipation inequality and the homogeneity conditions.

\section{Efficiency}

We consider a cyclic, power-producing process of a closed discrete system which works between two heat reservoirs of constant thermostatic temperatures $T_{H}^{*}>T_{L}^{*}$. The contact temperatures of the two contacts between the system and the reservoirs are $\Theta_{H}$ and $\Theta_{L}$, the heat exchanges through the inertial contacts are $\dot{Q}_{H}^{*}<0$ and $\dot{Q}_{L}^{*}>0$. According to the defining inequality (24), we obtain for the closed system

$$
\left(\frac{1}{T_{H}^{*}}-\frac{1}{\Theta_{H}}\right) \dot{Q}_{H}^{*} \geq 0, \quad\left(\frac{1}{T_{L}^{*}}-\frac{1}{\Theta_{L}}\right) \dot{Q}_{L}^{*} \geq 0
$$

These inequalities yield

$$
T_{H}^{*} \geq \Theta_{H}, \quad \Theta_{L} \geq T_{L}^{*}
$$

Integration over the cycle time results in

$$
\begin{gathered}
\oint \frac{\dot{Q}_{H}^{*}}{\Theta_{H}} d t \leq \frac{1}{T_{H}^{*}} \oint \dot{Q}_{H}^{*} d t=: \frac{1}{T_{H}^{*}} Q_{H}^{*}, \\
\oint \frac{\dot{Q}_{L}^{*}}{\Theta_{L}} d t \leq \frac{1}{T_{L}^{*}} \oint \dot{Q}_{L}^{*} d t=: \frac{1}{T_{L}^{*}} Q_{L}^{*} .
\end{gathered}
$$


We obtain with the mean value theorem

$$
\begin{gathered}
\oint \frac{\dot{Q}_{H}^{*}}{\Theta_{H}} d t=\frac{Q_{H}^{*}}{\left[\Theta_{H}\right]} \leq \frac{Q_{H}^{*}}{T_{H}^{*}} \quad \longrightarrow \quad T_{H}^{*} \geq\left[\Theta_{H}\right], \\
\oint \frac{\dot{Q}_{L}^{*}}{\Theta_{L}} d t=\frac{Q_{L}^{*}}{\left[\Theta_{L}\right]} \leq \frac{Q_{L}^{*}}{T_{L}^{*}} \quad \longrightarrow \quad\left[\Theta_{L}\right] \geq T_{L}^{*} .
\end{gathered}
$$

Here, the square brackets denote mean values over the cyclic process. A definition of a non-equilibrium efficiency is

$$
\eta_{\text {neq }}:=1-\frac{\left[\Theta_{L}\right]}{\left[\Theta_{H}\right]} \leq 1-\frac{T_{L}^{*}}{T_{H}^{*}}=\eta_{C A R} .
$$

The latter inequality stems from Eq. (75) and (76). The efficiency $\eta_{n e q}$ is a more realistic measure for non-equilibrium processes than the Carnot efficiency $\eta_{C A R}$, which belongs to reversible processes.

\section{Conclusion}

There are many different definitions of non-equilibrium temperatures [26]. The main point with respect to such definitions is to find a thermometer measuring this so defined non-equilibrium temperature. Here, in accordance with the second law, a non-equilibrium temperature, the contact temperature, and dynamic chemical potentials are phenomenologically introduced as contact quantities in comparison with the appropriate quantities of the contacting reservoir. As a result, these contact quantities can be measured directly by zeros of the related exchange quantities. The non-equilibrium entropy rate is integrable if the system is adiabatically unique. Because the contact temperature is independent of the internal energy, the Maxwell relations of the non-equilbrium entropy have a slightly different shape than those in equilibrium. As usual in continuum thermodynamics, also in the non-equilibrium theory of discrete systems, the dissipation inequality represents a constraint for the constitutive equations, a fact which is demonstrated for a simple material in non-equilibrium.

\section{Acknowledgments}

The author thanks the reviewers of the paper for their careful reading, their valuable remarks, and their questions and hints concerning the application of the contact quantities in future research. 


\section{Appendix}

Starting out with the second part of Eq. (22), we obtain

$$
\begin{aligned}
& \frac{1}{2}\left(\frac{\boldsymbol{\mu}}{\Theta}-\right.\left.\frac{\boldsymbol{\mu}^{*}}{T^{*}}\right) \cdot\left(\dot{\mathbf{n}}^{e *}-\dot{\mathbf{n}}^{e}\right)= \\
&= \frac{1}{2} \frac{1}{\Theta}\left(\boldsymbol{\mu}-\boldsymbol{\mu}^{*}\right) \cdot \dot{\mathbf{n}}^{e *}-\frac{1}{2} \frac{1}{T^{*}}\left(\boldsymbol{\mu}-\boldsymbol{\mu}^{*}\right) \cdot \dot{\mathbf{n}}^{e}+ \\
& \quad+\frac{1}{2}\left(\frac{1}{T^{*}}-\frac{1}{\Theta}\right) \boldsymbol{\mu} \cdot \dot{\mathbf{n}}^{e}-\frac{1}{2}\left(\frac{1}{T^{*}}-\frac{1}{\Theta}\right) \boldsymbol{\mu}^{*} \cdot \dot{\mathbf{n}}^{e *}= \\
&=\frac{1}{2}\left(\frac{1}{T^{*}}+\frac{1}{\Theta}\right)\left(\boldsymbol{\mu}-\boldsymbol{\mu}^{*}\right) \cdot \dot{\mathbf{n}}^{e *}-\frac{1}{2}\left(\frac{1}{T^{*}}-\frac{1}{\Theta}\right)\left(\boldsymbol{\mu}+\boldsymbol{\mu}^{*}\right) \cdot \dot{\mathbf{n}}^{e *} .
\end{aligned}
$$

The first part of Eq. (22) becomes

$$
\begin{aligned}
& \frac{1}{2}\left(\frac{1}{T^{*}}-\frac{1}{\Theta}\right)\left[\dot{Q}^{*}+\mathbf{h}^{*} \cdot \dot{\mathbf{n}}^{e *}-\dot{Q}-\mathbf{h} \cdot \dot{\mathbf{n}}^{e}\right]= \\
& \quad=\frac{1}{2}\left(\frac{1}{T^{*}}-\frac{1}{\Theta}\right)\left(\dot{Q}^{*}-\dot{Q}\right)+\frac{1}{2}\left(\frac{1}{T^{*}}-\frac{1}{\Theta}\right)\left(\mathbf{h}^{*}+\mathbf{h}\right) \cdot \dot{\mathbf{n}}^{e *}
\end{aligned}
$$

Inserting Eqs. (78) and (79) into Eq. (22) results in Eq. (23):

$$
\begin{gathered}
\Phi^{*}+\Phi=\frac{1}{2}\left(\frac{1}{T^{*}}-\frac{1}{\Theta}\right)\left(\dot{Q}^{*}-\dot{Q}\right)+\frac{1}{2}\left(\frac{1}{T^{*}}+\frac{1}{\Theta}\right)\left(\boldsymbol{\mu}-\boldsymbol{\mu}^{*}\right) \cdot \dot{\mathbf{n}}^{e *}+ \\
+\frac{1}{2}\left(\frac{1}{T^{*}}-\frac{1}{\Theta}\right)\left(T^{*} \mathbf{s}^{*}+\Theta \mathbf{s}\right) \cdot \dot{\mathbf{n}}^{e *}
\end{gathered}
$$

\section{References}

[1] Muschik, W., Why so many "schools" of thermodynamics?, Forsch. Ingenieurwes. 71 (2007), 149-161.

[2] Day, W.A., The Thermodynamics of Simple Materials with Fading Memory, Springer, Berlin, 1972.

[3] Šilhavý, M., The Mechanics and Thermodynamics of Continuous Media, Springer, Berlin, 1997.

[4] Jou, D., Casa-Vázquez, J., Lebon, G., Extended Irreversible Thermodynamics, Springer, Berlin, 1993.

[5] Müller, I., Ruggeri, T., Extended Thermodynamics, Springer, New York, 1993.

[6] Muschik, W., Survey of some branches of thermodynamics, J. Non-Equilib. Thermodyn., 33 (2008), 165-198.

[7] Wilmański, K., Thermomechanics of Continua, Springer, Berlin, 1998.

[8] Haupt, P., Continuum Mechanics and Theory of Materials, Springer, Berlin, 2000. 
[9] Maugin, G.A., The Thermomechanics of Nonlinear Irreversible Behaviors, An Introduction, World Scientific, Singapore, 1999.

[10] Kjelstrup, S., Bedeaux, D., Non-Equilibrium Thermodynamics of Heterogeneous Systems, World Scientific, Singapore, 2008.

[11] Hoffmann, K.H., Burzler, J.M., Schubert, S., Endoreversible thermodynamics, J. Non-Equilib. Thermodyn., 22 (1997), 311-355.

[12] Muschik, W., Domínguez-Cascante, R., On extended thermodynamics of discrete systems, Physica A, 233 (1996), 523-550.

[13] Muschik, W., Brunk, G., Temperatur und Irreversibilität in der Rationalen Mechanik, ZAMM, 55 (1975), T 102-T 105.

[14] Muschik, W., Empirical foundation and axiomatic treatment of non-equilibrium temperature, Arch. Rat. Mech. Anal., 66 (1977), 379-401.

[15] Muschik, W., Brunk, G., A concept of non-equilibrum temperature, Int. J. Eng. Sci., 15 (1977), 377-389.

[16] Muschik, W., Fundamentals of dissipation inequalities, I. Discrete systems, J. NonEquilib. Thermodyn., 4 (1979), 277-294.

[17] Muschik, W., Aspects of Non-Equilibrium Thermodynamics, section 4, World Scientific, Singapore, 1990.

[18] Muschik, W., Gümbel, S., Does Clausius' inequality analogue exist for open discrete systems?, J. Non-Equilib. Thermodyn., 24 (1999), 97-106.

[19] Muschik, W., Berezovski, A., Thermodynamic interaction between two discrete systems in non-equilibrium, J. Non-Equilib. Thermodyn., 29 (2004), 237-255.

[20] Muschik, W., Recent developments in nonequilibrium thermodynamics, in: Lecture Notes in Physics, Vol. 199, p. 387, Springer, Berlin, 1984.

[21] Muschik, W., Fundamentals of non-equilibrium thermodynamics, in: Non-Equilibrium Thermodynamics with Application to Solids, CISME Courses and Lectures 336, Ed. W. Muschik, pp. 1-63, Springer, Wien, 1993.

[22] Muschik, W., Aspects of Non-Equilibrium Thermodynamics, sect. 1.2, World Scientific, Singapore, 1990.

[23] Muschik, W., Aspects of Non-Equilibrium Thermodynamics, sect. 5.4, World Scientific, Singapore, 1990.

[24] Muschik, W., Papenfuss, C., Ehrentraut, H., A sketch of continuum thermodynamics, J. Non-Newtonian Fluid Mech., 96 (2001), 255-290, sect. 4.2.3.

[25] Kestin, J., A Course in Thermodynamics, Vol. II, sect. 21.4, Hemisphere, Washington, 1979.

[26] Casas-Vázquez, J., Jou, D., Temperature in non-equilibrium states: a review of open problems and current proposals, Rep. Prog. Phys., 66 (2003), 1937-2023.

Paper received: 2008-11-06

Paper accepted: $\quad$ 2008-12-09 